\title{
A Tight Lower Bound on the Classical Communication Cost of Entanglement Dilution
}

\author{
Aram W. Harrow, Student Member, IEEE, and Hoi-Kwong Lo, Member, IEEE
}

\begin{abstract}
Suppose two distant observers, Alice and Bob, share some form of entanglement-quantum correlations-in some bipartite pure quantum states. They may apply local operations and classical communication to convert one form of entanglement to another. Since entanglement is regarded as a resource in quantum information processing, it is an important question to ask how much classical communication, which is also a resource, is needed in the inter-conversion process of entanglement. In this paper, we address this important question in the many-copy case. The inter-conversion process of entanglement is usually divided into two types: concentrating the entanglement from many partially entangled states into a smaller number of maximally entangled states (i.e., singlets) and the reverse process of diluting singlets into partially entangled states.

It is known that entanglement concentration requires no classical communication, but the best prior art result for diluting to $N$ copies of a partially entangled state requires an amount of communication on the order of $\sqrt{N}$. Our main result is to prove that this prior art result is optimal up to a constant factor; any procedure for approximately creating $N$ partially entangled states from singlets requires $\Omega(\sqrt{N})$ bits of classical communication. Previously not even a constant bound was known for approximate entanglement transformations.

We also prove a lower bound on the inefficiency of the process: to dilute singlets to $N$ copies of a partially entangled state, the entropy of entanglement must decrease by $\Omega(\sqrt{N})$. Moreover, we introduce two new tools $-\delta$-significant subspaces and the standard form protocol reduction in entanglement manipulations. We hope that these two new tools will be useful in other work in quantum information theory.
\end{abstract}

Index Terms-Communication, entanglement dilution, entanglement transformations, quantum information.

\section{BACKGROUND}

$\mathbf{E}$ NTANGLEMENT-quantum correlations-is an important subject of study in quantum information theory. This paper deals with pure state bipartite entanglement only. A standard form of pure state entanglement shared by two (distant) parties, traditionally called Alice and Bob, is a maximally entangled state $|\Phi\rangle_{A B}=\frac{1}{\sqrt{2}}\left(|01\rangle_{A B}-|10\rangle_{A B}\right)$. It has the prop-

Manuscript received July 3, 2002; revised September 23, 2003. The work of A. W. Harrow was supported in part by the National Security Agency and the Advanced Research and Development Activity under Army Research Office Contract DAAD19-01-1-06. The work of H.-K. Lo was supported by CFI, CRC program, NSERC, OIT, and PREA. The material in this paper was presented at the IEEE International Symposium on Information Theory, Yokohama, Japan, June/July 2003.

A. W. Harrow is with the Department of Physics, Massachusetts Institute of Technology, Cambridge, MA 02139 USA (e-mail: aram@mit.edu).

H.-K. Lo is with the Department of Electrical and Computer Engineering and the Department of Physics, University of Toronto, Toronto, ON M5S 3G4, Canada (e-mail: hklo@comm.utoronto.ca).

Communicated by P. W. Shor, Associate Editor for Quantum Information Theory.

Digital Object Identifier 10.1109/TIT.2003.822597 erty that it cannot be written as a direct product of individual states of the two particles. (i.e., it is impossible to find two states, $|u\rangle$ and $|v\rangle$, such that $\left.|\Phi\rangle_{A B}=|u\rangle_{A} \otimes|v\rangle_{B}\right)$. For this reason, the state $|\Phi\rangle_{A B}$ is called entangled. Note that Alice can, by applying a local operation on her side only, convert the state $|\Phi\rangle_{A B}$ to $\left|\Phi^{\prime}\right\rangle_{A B}=\frac{1}{\sqrt{2}}\left(|00\rangle_{A B}+|11\rangle_{A B}\right)$. Other forms of entangled states exist. An example is the state $|\psi\rangle_{A B}=$ $a|00\rangle_{A B}+b|11\rangle_{A B}$ where $|a|>|b|>0$ and $|a|^{2}+|b|^{2}=1$. Such a state is called partially entangled.

We will study entanglement as a resource in quantum information theory, along with other resources such as classical or quantum communication. For a resource model to make sense, different forms of the same resource need to be inter-convertible or fungible in the asymptotic limit.

In [1], bipartite pure state entanglement was proposed as asymptotically fungible and thus a resource. Suppose two distant observers, Alice and Bob, share $n$ copies of some entangled state $\left|\psi_{1}\right\rangle_{A B}$. They would like to apply local operations and classical communications (LOCC) to convert them into $k$ copies of another state $\left|\psi_{2}\right\rangle_{A B}$. They ask the question: what is the maximal value of the ratio $k / n$ in the limit of large $n$ such that the fidelity goes to one? (For the specific case where $\left|\psi_{1}\right\rangle_{A B}$ is a partially entangled state and $\left|\psi_{2}\right\rangle_{A B}$ is a maximally entangled state, the process is called entanglement concentration. If, instead, $\left|\psi_{1}\right\rangle_{A B}$ is a maximally entangled state and $\left|\psi_{2}\right\rangle_{A B}$ is a partially entangled state, the process is called entanglement dilution.)

Reference [1] proved that this optimal asymptotic ratio is given by the ratio of their entropies of entanglement: $E\left(\left|\psi_{1}\right\rangle_{A B}\right) / E\left(\left|\psi_{2}\right\rangle_{A B}\right)$. (Given a bipartite pure state $|\psi\rangle_{A B}$, its entropy of entanglement $E\left(|\psi\rangle_{A B}\right)$ is defined as the von Neumann entropy of Alice's (or Bob's) reduced state, i.e., $E\left(|\psi\rangle_{A B}\right)=S\left(\operatorname{Tr}_{B}|\psi\rangle\langle\psi|\right)$.) The proof is based on constructing asymptotically efficient LOCC procedures for entanglement concentration and dilution. However, these are only asymptotically reversible: in each direction we accept some inefficiency (so that instead of converting $\left|\Phi_{2}\right\rangle^{\otimes n E}$ to $|\psi\rangle^{\otimes n}$, we need to start with $\left|\Phi_{2}\right\rangle^{\otimes[n E+o(n)]}$ ) and a small error $\epsilon \in o(1)$ (so instead of obtaining the state $|\psi\rangle^{\otimes n}$, we get $\rho$ with $\left.\operatorname{Tr}\left|\rho-(|\psi\rangle\langle\psi|)^{\otimes n}\right|<\epsilon\right)$.

Furthermore, it was emphasized in [14], [16] that in some settings, the classical communication used in the inter-conversion process should be treated as a limited resource. For instance, in a well-known process called superdense coding [4], Alice and Bob, who share a maximally entangled pair, can encode two classical bits of information by sending only a single qubit (quantum bit or a two-level quantum system) from one to another. Since entanglement concentration can be performed with 
no classical communication [1], Alice and Bob could perform superdense coding just as effectively if they started with partially entangled states.

However, as noted in [16], the original process for entanglement dilution proposed in [1] requires $\Omega(n)$ bits of communication to create $n$ copies of a partially entangled state from singlets. For some applications, such as generating entanglement from a bipartite unitary interaction ([2], [7]) this cost would affect the optimal rate. Fortunately, $[16]$ also showed that the original dilution protocol in [1] could be modified to require only $o(n)$ bits of classical communication. Thus, even taking into account the cost of classical communication, any two states with the same entropy of entanglement are asymptotically interconvertible and entanglement remains a fungible resource.

The specific dilution procedure of [16] used $O(\sqrt{n})$ bits, but left open the question of whether this could be improved. The main result of this paper proves that no such improvement is possible. In other words, we will show that $\Omega(\sqrt{n})$ bits of classical communication are necessary for entanglement dilution and the result in [16] is, in fact, optimal up to a constant factor. Previously it was known that some exact entanglement transformations were only possible with a nonzero amount of classical communication, but for approximate transformations no bounds were known.

In addition to the question of classical communication cost, we also address the question of inefficiency in entanglement diluation. In order to create $n$ partially entanged states, each of entanglement $E$, with a fidelity close to one, we show that Alice and Bob must start with $n E+\Omega(\sqrt{n})$ singlets. In other words, $\Omega(\sqrt{n})$ singlets must be wasted in entanglement dilution.

A main motivation of our study is to understand fluctuations in a finite system [17]. For entanglement manipulation, these take the form of probability of failure, imperfect fidelity, suboptimal yield, and required classical communication. Given that any physical system must have only a finite number of constituents, it is important to understand the magnitude and origin of those problems. That is, how quickly does a finite-copy system approach its thermodynamic/asymptotic limit as its number of copies increases? As we will see later, some of those problems are related to the fact that when different states are not related to each other in trivial ways, we can only approximate them. Others are related to the spectrum of the Schmidt coefficients.

Errors and the probability of failure are results of discretization and can be made exponentially small in $n$. In contrast, we will show that the inefficiency and the classical communication cost of entanglement dilution are necessarily $\Omega(\sqrt{n})$ because they stem from differences in the shapes of spectra of the Schmidt coefficients of different states.

We will make this notion precise later, by defining the significant subspace (or $\delta$-significant subspace) of a density matrix $\rho$ to be a subspace that contains at least $\delta$ of the weight of $\rho$ for some $O(1)$ constant $\delta .{ }^{1}$ This definition generalizes typical subspaces, which are usually defined as containing almost all the weight of a density matrix.

\footnotetext{
${ }^{1}$ The idea of a $\delta$-significant subspace has a classical analog. In [6, Ch. 3], the idea of a high probability set for a distribution is described. We thank Debbie Leung for bringing this point to our attention.
}

We will show that, for a generic $\rho$ that is neither pure nor maximally mixed, the ratio of the size of a typical subspace of $\rho^{\otimes n}$ to that of a $\delta$-significant subspace (say, with $\delta=1 / 4$ ) of $\rho^{\otimes n}$ is large, namely, $2^{\Omega(\sqrt{n})}$. It is the logarithm of this ratio that gives rise to the fundamental constraint - that the inefficiency and classical communication cost of entanglement dilution have lower bounds of $\Omega(\sqrt{n})$. We hope that the $\delta$-significant subspace idea will be of utility in other work in quantum information theory.

Another new tool that we will introduce in this paper is the standard form protocol reduction (in Section III) in entanglement manipulation. We show that any strategy for entanglement manipulation of a bipartite pure state that involves $c$ bits of classical communication can equivalently be implemented by one that involves only 1) a single generalized measurement by Alice, which generates a $c$-bit outcome, 2) the transmission of the $c$-bit outcome to Bob, followed by 3) Bob performing a unitary transformation $U_{k}$ conditioned on this outcome, and 4) both sides discarding ancillary systems. This protocol reduction thus generalizes the protocol reduction theorem of [17] by accounting for the classical communication cost

To put these results in perspective, it is worth noting that the entanglement dilution and concentration protocols of [1] both reduce the entropy of entanglement by $O(\sqrt{n})$. In both cases, this amount of inefficiency turns out to be optimal. We will prove the dilution bound in Section V-A, and for concentration, [17] proved that $o(1)$ error requires an inefficiency of $\Omega(\sqrt{n}) .^{2}$ Similarly, both classical and quantum data compression require $\Omega(\sqrt{n})$ more space (either bits or qubits) to compress a source than would be implied by the entropy of the source. ${ }^{3}$

This paper is organized as follows. Section II contains the formal statement of our main result- that entanglement dilution necessarily requires $\Omega(\sqrt{n})$ classical bits of communication and an inefficiency of $\Omega(\sqrt{n})$. In Sections II and IV, we present useful intermediate results: in Section III, we show that a general strategy of entanglement dilution can be equivalently rephrased as a much simpler one. This is the standard form protocol reduction idea that we mentioned earlier. In Section IV, we define $\delta$-significant subspaces and study their properties. Our main result is proved in Section V and Section VI contains some concluding remarks and discussions.

\section{Statement of ThE Main Result}

If a partially entangled bipartite pure state $|\psi\rangle$ has entropy of entanglement $E$, then $|\psi\rangle^{\otimes n}$ can be approximately prepared by two distant parties, Alice and Bob, from roughly $n E$ singlets using only LOCC. The main result of the present paper is that any such dilution procedure must use $\Omega(\sqrt{n})$ bits of classical communication. Along the way, we will also prove that dilution protocols cannot be perfectly efficient, and inevitably waste

\footnotetext{
${ }^{2}$ This bound applies only to concentration protocols that create a deterministic number of singlets. If we relax this assumption, then $n$ states with entanglement $E$ can be converted to $m$ singlets where $m$ is a random variable with expectation $n E-O(\log n)$ [12]. For dilution, however, our $\Omega(\sqrt{n})$ bound applies equally well to variable yield dilution protocols since the proof is highly insensitive to the protocol's success probability.

${ }^{3}$ This well-known result is also a consequence of our Proposition 6.
} 
$\Omega(\sqrt{n})$ bits of entanglement. The formal statement of our main result is as follows.

Theorem 1: Let $|\psi\rangle \in \mathcal{H}_{A} \otimes \mathcal{H}_{B}$ be a bipartite pure state that is neither separable nor maximally entangled with its entropy of entanglement $E=S\left(\operatorname{Tr}_{B}|\psi\rangle\langle\psi|\right)$. Let $\left|\Phi_{d}\right\rangle=\frac{1}{\sqrt{d}} \sum_{i=1}^{d}|i\rangle|i\rangle$ be a maximally entangled state of dimension $d$. Then there exist a universal constant $\epsilon_{0}$ and constants $\alpha$ and $n_{0}$ that depend on $|\psi\rangle$ such that $\forall \epsilon \leq \epsilon_{0}, \forall n \geq n_{0}$, any entanglement dilution protocol transforming $\left|\Phi_{d}\right\rangle$ into $|\psi\rangle^{\otimes n}$ with error $\epsilon$, probability of success $2^{-s}$ and using $c$ bits of classical communication must have

a) $\log d \geq n E+\alpha \sqrt{n}$ and

b) $c+s \geq \alpha \sqrt{n}$.

This establishes the lower bounds as strongly as possible, by requiring only a constant bound on the error and disallowing the possibility of a tradeoff between inefficiency and classical communication cost. In Theorem 1 , we have made use of the following definitions.

Definition of the Trace Distance: The trace distance is defined as

$$
D(\rho, \sigma)=\operatorname{Tr}|\rho-\sigma|
$$

We will often use the equivalent formulation

$$
D(\rho, \sigma)=2 \max _{P} \operatorname{Tr}(P(\rho-\sigma))
$$

where $P$ is a projector [9]. It is important not to confuse this distance with the matrix norm, which we define in the usual way.

Definition of the Norm of a Matrix: By the norm of a matrix $A$, denoted $\|A\|$, we mean its largest singular value. Equivalently, $\|A\|=\sup _{|v|=1}|A v|$.

Definition of Error: By error $\epsilon$ we mean that upon success the protocol outputs not $|\psi\rangle^{\otimes n}$ but some possibly mixed state $\sigma$ with $D\left(\sigma,|\psi\rangle^{\otimes n}\right)=\epsilon$. For this proof, we require only that the protocol has an error $\epsilon$ smaller than a universal constant $\epsilon_{0} \approx$ 0.01 .

Definition of Failure: Failure, on the other hand, means that sometimes Alice's measurement yields a state that is far from $|\psi\rangle^{\otimes n}$.

Remark: Our lower bound on classical communication, stated in Part b) of Theorem 1, still holds even if the probability of a protocol succeeding is vanishingly small (i.e., $2^{-o(\sqrt{n})}$ ). At first glance, this might seem to be a surprisingly strong result. Why should our bound apply so broadly to probabilistic protocols? When we prove Theorem 1 (in Section V) we will find that the communication bound is independent of the amount of prior entanglement used. As a result, it is possible to convert any probabilistic protocol into a nearly deterministic protocol and vice versa in the following manner.

Suppose there exists a protocol with a $2^{-s}$ probability of success and $c$ bits of classical communication. We will show that it is always possible to perform a modified protocol with $1-\epsilon$ probability of success and $c+s+O(\log \log (1 / \epsilon))$ bits of communication, at the cost of massively increasing the inefficiency (i.e., the loss of entanglement). To implement the modified protocol, Alice performs her measurement $2^{s} O(\log 1 / \epsilon)$ times on different inputs and with $\approx 1-\epsilon$ probability she will succeed on at least one of them. She can then transmit the index of the successful block in $s+O(\log \log (1 / \epsilon))$ bits and send the $c$-bit measurement result corresponding only to the successful outcome while discarding the failures.

Conversely, $c$ bits of classical communication can always be eliminated by having Bob guess the message Alice would have sent with a $2^{-c}$ probability of success. (In this way, Alice needs to send Bob at most one additional bit to inform him whether his guess is correct.) Thus, it is, in general, impossible to bound either success probability or classical communication cost independently. Instead, Part b) of Theorem 1 gives a tradeoff between success probability and classical communication cost.

\section{REDUCING ENTANGLEMENT MANIPULATION PROTOCOLS TO A STANDARD FORM}

A general strategy for entanglement manipulation may involve two-way classical communication between Alice and Bob. Suppose Alice and Bob have some method of performing an approximate entanglement manipulation procedure using local operations and $c$ total bits of classical communication (in either direction). In entanglement dilution, for example, Alice and Bob begin sharing some number of perfect singlets and with high probability end with a mixed state that approximates many copies of a partially entangled state. In this section, we will simplify the description of any such entanglement manipulation procedure.

Claim 2: Given a pure bipartite initial state and any LOCC entanglement manipulation protocol by Alice and Bob that involves no more than $c$ bits of classical communication, there exists an equivalent strategy (meaning it uses the same amount of communication, takes the same inputs, and has the same output distribution) consisting of the following steps.

1) Alice performs a generalized measurement $\left\{M_{k}\right\}$ with no more than $2^{c}$ outcomes on her half of the input.

2) She transmits the result to Bob using $c$ bits of classical communication.

3) Bob performs a unitary operation $U_{k}$ conditioned on the result.

4) Both sides discard ancillary systems.

A similar claim was proved in [17]: it was shown that any LOCC entanglement manipulation strategy that begins and ends with pure states can be reduced to one that uses only one-way communication.

However, we need to address two new subtleties here. First, we need to consider approximate entanglement transformations which begin in pure states but can end in mixed states. Second, [17] does not specifically address the issue of classical communication cost. In our modified protocol we need Alice to transmit her entire measurement outcome using no more classical communication than the original protocol.

Proof: Local operations can be broken into unitary transforms, measurements, adding ancilla systems, and discarding 

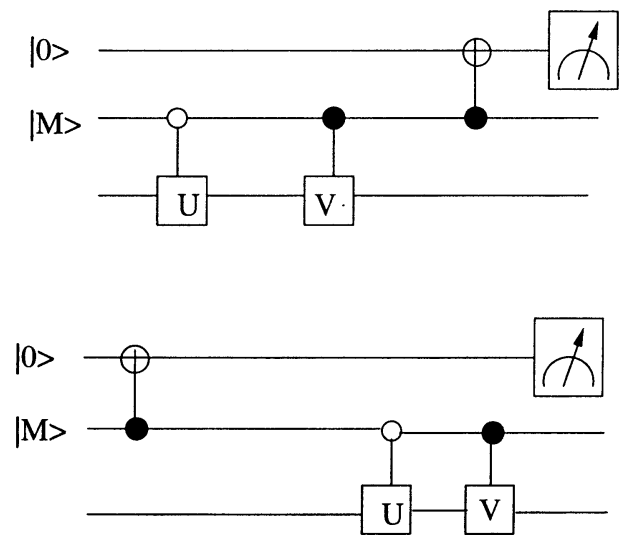

Fig. 1. Demonstration that $\$$ commutes with the gate in (3). The top line is an ancilla used to illustrate how $\$$ can be performed, the middle line holds the measurement outcome $|M\rangle$, and the bottom line represents the rest of the system which we act on. Since the two circuits are equivalent, it follows that $\$$ commutes with any gates of the form in (3).

ancillas [21]. Without loss of generality, we can add all the ancilla systems at the beginning of the protocol and discard all the subsystems at the end.

Simplifying measurements and classical communication is more complicated. Consider any measurement performed in a dilution protocol. By Neumark's theorem [20], we can convert such a measurement into a unitary operation followed by a projective measurement in the computational basis. This can be thought of as coupling the system to a measurement apparatus and then recording the state of the measuring device. Denote the effects of a projective measurement on a single qubit by a superoperator $\$$. It has Kraus operators $|0\rangle\langle 0|$ and $|1\rangle\langle 1|$. We can also represent the classical channel between Alice and Bob as a quantum channel that maps input $\rho$ to output $\$(\rho)$.

At this point, the most general protocol is equivalent to one in which Alice performs a unitary operation, applies $\$$ some number of times, and transmits some bits via the classical channel; then Bob performs some unitary operation conditioned on the message, applies $\$$ and sends some qubits through the classical channel; and so on. Conditioning a unitary operation on a measurement outcome $M$ can be written as a single unitary matrix

$$
|0\rangle\langle 0|\otimes U+| 1\rangle\langle 1| \otimes V
$$

where $U$ and $V$ are unitary gates operating on the target system. Since we never rewrite the qubits storing measurement outcomes and only use them for classical control of the rest of the system, the only gates we will apply to them will be of the form in (3). From Fig. 1, it is easy to see that these commute with $\$$.

Thus, without loss of generality we can defer every application of $\$$ until the end of the protocol.

This seemingly trivial step turns out to be equivalent to requiring Alice and Bob to communicate their complete measurement outcomes. To see this, note that until the final discarding of ancillas, $\$$ is only applied to the bits that Alice and Bob actually send to each other. Equivalently, Alice and Bob only perform projective measurements on qubits and they always report their measurement outcomes. This means that both parties always know the joint state exactly until the final step when they discard ancillas. For this reason, we can deal with pure states only, a situation sometimes referred as the Church of the Larger Hilbert space. ${ }^{4}$

Under these conditions, [17] showed that any measurement performed by Bob where he communicates the outcome to Alice can be simulated by a measurement by Alice where she communicates the outcome to Bob (without changing classical communication cost). This result holds because the Schmidt decomposition of a pure state is always symmetric under the interchange of Alice and Bob. Therefore, without loss of generality, we can consider a reduced protocol where only Alice performs measurements and at the end communicates the entire measurement record to Bob.

As a final simplification, we note that Alice can combine a whole sequence of measurements into a single measurement.

In summary, given any fixed protocol for entanglement manipulation, we can construct an equivalent reduced protocol that consists of the following steps: a generalized measurement by Alice, transmission of the complete measurement outcome to Bob, a unitary operation by Bob conditioned on the measurement, and then discarding of ancillas on both sides. Moreover, the modified protocol uses the same amount of classical communication as the original protocol.

\section{Significant Subspaces of A Density Matrix}

A typical subspace of a density matrix $\rho$ is a vector space that contains most (i.e., $1-o(1)$ ) of the weight of $\rho$ but in general has a dimension much smaller than the rank of $\rho[6]$. For our proof, we will introduce the related concept of a significant subspace, which is a vector space that contains a significant portion (meaning some $O(1)$ constant) of the weight of $\rho$. Neither significant subspaces nor typical subspaces have properties that are unique to quantum information theory, but we will find it more convenient to state our definitions in terms of density matrices rather than probability distributions.

Definition of a Significant Subspace: Let $\Pi$ be the projector onto a finite-dimensional vector space $V$. For any density matrix $\rho$ and $0 \leq \delta \leq 1$, we say that $V$ is a significant subspace of $\rho$ (or a $\delta$-significant subspace) if $\operatorname{Tr} \Pi \rho \geq \delta$.

For any $\delta$ there can be many different significant subspaces and the only upper bound we can place on their dimension is rank $\rho$. However, we will find it useful to examine the minimum dimension of any $\delta$-significant subspace for a matrix $\rho$. Denote this dimension by $\mathcal{S}(\rho, \delta)$ and define it by

$$
\mathcal{S}(\rho, \delta)=\left\{\min \operatorname{Tr} \Pi \mid \Pi^{2}=\Pi, \operatorname{Tr} \Pi \rho \geq \delta\right\} .
$$

In discussing bipartite entanglement, the rank of a density matrix is often useful to work with because it corresponds to the Schmidt number of an entangled state, which can never increase under LOCC. Unfortunately, in general, a small perturbation can change the rank by an arbitrary amount. Significant subspaces are more stable under perturbation, and we can use $\mathcal{S}(\rho, \delta)$ to derive robust bounds on the rank.

\footnotetext{
${ }^{4}$ The Church of the Larger Hilbert space is a fruitful idea in quantum information theory. It has been applied in, for example, the proof of the impossibility of quantum bit commitment [15], [18] and quantum oblivious transfer [13]. For a review, see, for example, [5].
} 
Proposition 3: If $0 \leq \delta \leq 1$ and $\rho, \sigma$ are density matrices with $D(\rho, \sigma) \leq 2(1-\delta)$ then rank $\sigma \geq \mathcal{S}(\rho, \delta)$

Proof: Let $P$ project onto the support of $\sigma$. Then $\operatorname{Tr} P=$ rank $\sigma$ and $\operatorname{Tr} P \sigma=1$. Using (2), we find

$$
2(1-\delta) \geq D(\rho, \sigma) \geq 2 \operatorname{Tr} \Pi(\sigma-\rho)
$$

for any projector $\Pi$. Combining this with $\operatorname{Tr} P \sigma=1$, we obtain $\operatorname{Tr} P \rho \geq \delta$. From the definition of $\mathcal{S}$ we have that

$$
\mathcal{S}(\rho, \delta) \leq \operatorname{Tr} P=\operatorname{rank} \sigma .
$$

Significant subspaces are also preserved reasonably well under tensor product.

Proposition 4: Let $A$ and $B$ be density matrices with $\delta_{A}, \delta_{B} \geq 0$ and $\delta_{A}+\delta_{B} \leq 1$. Then

$$
\begin{aligned}
\mathcal{S}\left(A \otimes B, \delta_{A}+\delta_{B}\right) & \geq \mathcal{S}\left(A \otimes B, \delta_{A}+\delta_{B}-\delta_{A} \delta_{B}\right) \\
& >\left(\mathcal{S}\left(A, \delta_{A}\right)-1\right)\left(\mathcal{S}\left(B, \delta_{B}\right)-1\right) .
\end{aligned}
$$

Remark: Proposition 4 applies to a density matrix that is a product of mixtures. Note that this is a more restricted condition than the requirement that the state is separable, which would have required only that the state be a mixture of products.

Proof: Let $a=\mathcal{S}\left(A, \delta_{A}\right)-1$ and $b=\mathcal{S}\left(B, \delta_{B}\right)-1$. Define a projector $\Pi_{A}$ that projects onto the $a$ eigenvectors of $A$ with the highest eigenvalues and likewise define $\Pi_{B}$ to project onto the $b$ eigenvectors of $B$ with the highest eigenvalues. From the definition of $\mathcal{S}$, we know that $\operatorname{Tr} \Pi_{A} A<\delta_{A}$ and $\operatorname{Tr} \Pi_{B} B<\delta_{B}$.

Now consider the orthogonal complements of $\Pi_{A}$ and $\Pi_{B}$. Every eigenvalue of $\left(\mathbb{1}-\Pi_{A}\right) A$ has at least $a$ eigenvalues of $A$ greater than or equal to it. Likewise, every eigenvalue of $(\mathbb{1}-$ $\left.\Pi_{B}\right) B$ has at least $b$ eigenvalues of $B$ greater than or equal to it. Therefore, every eigenvalue of

$$
\left(\left(\mathbb{1}-\Pi_{A}\right) \otimes\left(\mathbb{1}-\Pi_{B}\right)\right)(A \otimes B)
$$

has at least $a b$ eigenvalues of $A \otimes B$ greater than or equal to it. Equivalently, the $a b$ highest eigenvalues of $A \otimes B$ correspond to eigenvectors in the support of $\mathbb{1}-\left(\mathbb{1}-\Pi_{A}\right) \otimes\left(\mathbb{1}-\Pi_{B}\right)$.

Furthermore

$$
\begin{aligned}
\operatorname{Tr}(\mathbb{1}- & \left.\left(\mathbb{1}-\Pi_{A}\right) \otimes\left(\mathbb{1}-\Pi_{B}\right)\right)(A \otimes B) \\
& =\operatorname{Tr} \Pi_{A} A+\operatorname{Tr} \Pi_{B} B-\operatorname{Tr} \Pi_{A} A \operatorname{Tr} \Pi_{B} B \\
& <\delta_{A}+\delta_{B}-\delta_{A} \delta_{B} .
\end{aligned}
$$

In the above, we make use of the fact that $\operatorname{Tr} \Pi_{A} A<\delta_{A}$, $\operatorname{Tr} \Pi_{B} B<\delta_{B}$, and that $\operatorname{Tr} \Pi_{A} A+\operatorname{Tr} \Pi_{B} B-\operatorname{Tr} \Pi_{A} A \operatorname{Tr} \Pi_{B} B$ is an increasing function in both $\operatorname{Tr} \Pi_{A} A$ and $\operatorname{Tr} \Pi_{B} B$.

Thus, the largest $a b$ eigenvectors of $A \otimes B$ have weight less than $\delta_{A}+\delta_{B}-\delta_{A} \delta_{B}$. This implies

$$
a b<\mathcal{S}\left(A \otimes B, \delta_{A}+\delta_{B}-\delta_{A} \delta_{B}\right) \leq \mathcal{S}\left(A \otimes B, \delta_{A}+\delta_{B}\right)
$$

the desired result.

One application of typical subspaces is to show that $\rho^{\otimes n}$ can be compressed to $2^{n S(\rho)+O(\sqrt{n})}$ dimensions with asymptotically small error. This is because for large $n$, the spectrum of $\rho^{\otimes n}$ (for any $\rho$ ) approaches a Gaussian distribution and almost all eigenvalues are between $2^{-n E-O(\sqrt{n})}$ and $2^{-n E+O(\sqrt{n})}[6]$.

To prove that improving upon this result is impossible, we will examine the significant subspaces of $\rho^{\otimes n}$.

First we will need to state central limit theorem in a manner that bounds the rate of convergence to Gaussianity.

Lemma 5 (Berry-Esseén): Let $\rho$ have eigenvalues $p_{1}, \ldots, p_{d}$ and define

$$
E=-\sum_{i} p_{i} \log p_{i}, \quad \alpha^{2}=\sum_{i} p_{i}\left(\log p_{i}+E\right)^{2}
$$

(with $\alpha>0$ ), and

$$
\beta=\sum_{i} p_{i}\left|\log p_{i}+E\right|^{3} .
$$

Let $\mu(a, b)$ denote the sum of all eigenvalues of $\rho^{\otimes n}$ between $2^{a}$ and $2^{b}$. Then, for all $a \leq b$

$$
\left|\mu(a, b)-N\left(\frac{a+n E}{\sqrt{n} \alpha}, \frac{b+n E}{\sqrt{n} \alpha}\right)\right|<\frac{25 \beta}{\sqrt{n}}
$$

where $N\left(x_{1}, x_{2}\right)$ is the cumulative normal distribution

$$
N\left(x_{1}, x_{2}\right) \equiv \frac{1}{\sqrt{2 \pi}} \int_{x_{1}}^{x_{2}} e^{-\frac{x^{2}}{2}} d x .
$$

Proof: A proof can be found in [3].

As a corollary, for any $\rho$ and $\delta$ there exists $n_{0}$ such that for $n \geq n_{0}$ the left side of (8) is less than $\delta$. Another useful consequence is that $n$ copies of a state with entropy $E$ have significant subspaces of dimension $2^{n E \pm O(\sqrt{n})}$.

Proposition 6: Let $\rho, E, \alpha$, and $\beta$ be as in Lemma 5. Then there exist $\delta<1, C, n_{0}$ such that $\mathcal{S}\left(\rho^{\otimes n}, \delta\right)>C 2^{n E+\alpha \sqrt{n}}$ for all $n \geq n_{0}$.

Here $\delta$ and $C$ are universal constants and $n_{0}$ depends only on $\beta$; one valid choice would be $\delta=0.95, C=0.01$. and $n_{0}=10^{7} \beta^{2}$.

Proof: Let $P$ be the projector onto the eigenvectors of $\rho^{\otimes n}$ with eigenvalues above $2^{-n E-1.1 \alpha \sqrt{n}}$. Applying Lemma 5 yields

$$
\operatorname{Tr} P \rho^{\otimes n} \leq N(-1.1, \infty)+\frac{25 \beta}{\sqrt{n}} \approx 0.94+\frac{25 \beta}{\sqrt{n}}
$$

which for some choice of $n_{0}$ and $\delta$ can be guaranteed to be less than $\delta$. Since $P$ picks out the largest eigenvectors, it minimizes the dimension of a $\delta$-significant subspace. Therefore, we have $\mathcal{S}\left(\rho^{\otimes n}, \delta\right)>\operatorname{Tr} P$. Now, to lower-bound $\operatorname{Tr} P$, note that the weight of eigenvalues of $\rho^{\otimes n}$ between $2^{-n E-1.1 \alpha \sqrt{n}}$ and $2^{-n E-\alpha \sqrt{n}}$ is at least $N(-1.1,-1)-\frac{25 \beta}{\sqrt{n}}$ which we can make greater than some constant $C>0$. Since the eigenvalues in this region are no greater than $2^{-n E-\alpha \sqrt{n}}$ it follows that

$$
C 2^{n E+\alpha \sqrt{n}} \leq \operatorname{Tr} P \leq \mathcal{S}\left(\rho^{\otimes n}, \delta\right) .
$$

Remark: Proposition 6 can be generalized to show that for any $r>0$, there exist $\delta_{r}<1, C$, and $n_{0}$ such that $\mathcal{S}\left(\rho^{\otimes n}, \delta_{r}\right)>$ $C 2^{n E+r \alpha \sqrt{n}}$ for all $n \geq n_{0}$.

We are now ready to prove our main result. 


\section{PRoOF OF THE MAIN THEOREM}

\section{A. The Inefficiency Bound}

Combining Proposition 3 with Proposition 6, we find that any state $\sigma$ with $D\left(\sigma, \rho^{\otimes n}\right)<2(1-\delta)$ must have support on a space of dimension $\Omega\left(2^{n E+\alpha \sqrt{n}}\right) .5$

This result allows us to prove that the inefficiency (loss of entanglement) bound applies to any dilution protocol.

Proof: [of Part a) of Theorem 1] Here we will not need the protocol reduction of Section III. Instead, represent a general quantum operation as a map from pure states to ensembles of pure states. For example, the $\$$ operation of Section III can be said to map the pure state $a|0\rangle+b|1\rangle$ to the ensemble $\left\{p_{i},|i\rangle\right\}_{i=0,1}$ with $p_{0}=|a|^{2}$ and $p_{1}=|b|^{2}$. Now suppose that Alice and Bob start with $\left|\Phi_{d}\right\rangle$, obtain a series of measurement outcomes that indicate the protocol has succeeded, and end with an ensemble of states $\left\{p_{i},\left|\varphi_{i}\right\rangle\right\}$. By "success" we mean that their resulting density matrix is close to the desired state; i.e., $D\left(\sum_{i} p_{i}\left|\varphi_{i}\right\rangle\left\langle\varphi_{i}|,| \psi\right\rangle^{\otimes n}\right)<\epsilon$.

Since the Schmidt number never has any chance of increasing, we must have $\operatorname{Sch}\left|\varphi_{i}\right\rangle \leq d$ for all $i$. Furthermore, since the trace distance is convex, there exists an $i$ for which $D\left(\left|\varphi_{i}\right\rangle,|\psi\rangle^{\otimes n}\right)<\epsilon$. Let $\rho=\operatorname{Tr}_{B}|\psi\rangle\langle\psi|$, let $E, \alpha$ and $\beta$ be as in Lemma 5, and choose $\delta, C$, and $n_{0}$ according to Proposition 6 . Then choose $\epsilon_{0}$ such that $\epsilon_{0}<2(1-\delta)$. Since tracing out Bob's system cannot increase the trace distance, we have

$$
D\left(\operatorname{Tr}_{B}\left|\varphi_{i}\right\rangle\left\langle\varphi_{i}\right|, \rho^{\otimes n}\right)<\epsilon<2(1-\delta) .
$$

Again we apply Propositions 3 and 6 to find that

$$
C 2^{n E+\alpha \sqrt{n}} \leq \operatorname{rank} \operatorname{Tr}_{B}\left|\varphi_{i}\right\rangle\left\langle\varphi_{i}|=\operatorname{Sch}| \varphi_{i}\right\rangle \leq d
$$

proving the desired result (up to an overall constant that we can absorb into $\alpha$ and $n_{0}$ ).

\section{B. The Communication Bound}

In Section III, we reduced an arbitrary dilution protocol to one consisting of a generalized measurement by Alice, a local unitary by Bob conditioned on the result, and then the discarding of ancilla systems by both parties.

Alice's measurement is the interesting step, since we can relate the spectrum of the measurement operator to the Schmidt coefficients of the resulting state. If the final state roughly resembles $|\psi\rangle^{\otimes n}$ then the measurement outcome that produced it must also have a typical subspace (say of weight 0.99 ) with dimension $2^{\Omega(\sqrt{n})}$ times larger than some significant subspace (of weight around 0.25$)$. We will show that any such measurement outcome occurs with probability $2^{-\Omega(\sqrt{n})}$ and thus that $\Omega(\sqrt{n})$ bits of communication are necessary to tell Bob the result of Alice's measurement.

To deal with the complication of discarding ancillas, we state one final lemma.

\footnotetext{
${ }^{5}$ This means that to guarantee $O(1)$ error, Schumacher compression (and hence the dilution protocol given by [1]) must have $\Omega(\sqrt{n})$ inefficiency. In Section V-A, we will use a similar argument to prove that this inefficiency bound applies to any dilution protocol.
}

Lemma 7: Consider a bipartite Hilbert space $\mathcal{H}_{A} \otimes \mathcal{H}_{B}$ with states $|\psi\rangle \in \mathcal{H}_{A} \otimes \mathcal{H}_{B},|\phi\rangle \in \mathcal{H}_{A}$ and

$$
D\left(\operatorname{Tr}_{B}|\psi\rangle\langle\psi|,| \phi\rangle\langle\phi|\right)<\epsilon
$$

Then, $\exists|\gamma\rangle \in \mathcal{H}_{B}$ such that

$$
D(|\psi\rangle,|\phi\rangle \otimes|\gamma\rangle)<2 \epsilon .
$$

In other words, if tracing out $\mathcal{H}_{B}$ leaves the state almost pure, then the subsystems must have been almost separable to begin with. This is proved in the Appendix.

Now we can proceed with the proof of the classical communication bound.

Proof: [of part b) of Theorem 1] By Claim 2, it suffices to consider an entanglement dilution procedure with the following form.

Alice first performs a generalized measurement $\left\{M_{k}\right\}$ with $2^{c}$ outcomes on her half of $\left|\Phi_{d}\right\rangle$ and transmits her $c$-bit measurement outcome $k$ to Bob, who performs some unitary operation $U_{k}$ conditioned on the result. The result of the measurement is $k$ with probability $\frac{1}{d} \operatorname{Tr} M_{k} M_{k}^{\dagger}$, leaving Alice and Bob with the pure state $\left|x_{k}\right\rangle_{A A^{\prime} B B^{\prime}}$, which can be written as

$$
\left(M_{k} \otimes U_{k}\right)\left|\Phi_{d}\right\rangle=\sqrt{\frac{\operatorname{Tr} M_{k} M_{k}^{\dagger}}{d}}\left|x_{k}\right\rangle .
$$

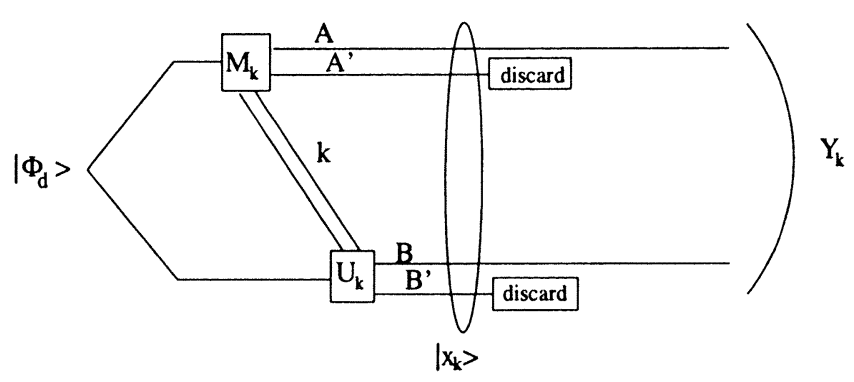

Then Alice and Bob trace out subsystems $A^{\prime}$ and $B^{\prime}$, leaving the state $Y_{k}=\operatorname{Tr}_{A^{\prime} B^{\prime}}\left|x_{k}\right\rangle\left\langle x_{k}\right|$. When the protocol succeeds (which occurs with probability $\geq 2^{-s}$ ), then $Y_{k}$ should be close to the desired state $|\psi\rangle^{\otimes n}$. Specifically, we should have

$$
D\left(Y_{k},|\psi\rangle^{\otimes n}\right)<\epsilon .
$$

Since there are at most $2^{c}$ successful values of $k$ (i.e., measurement outcomes for which (13) holds), there must exist at least one successful value of $k$ that has probability $\geq 2^{-(c+s)}$ of occuring. Equivalently

$$
\operatorname{Tr} M_{k} M_{k}^{\dagger} \geq \frac{d}{2^{c+s}}
$$

We will use this value of $k$ implicitly for the rest of the proof, and refer to $M, U,|x\rangle, Y$, and so forth instead of $M_{k}, U_{k},\left|x_{k}\right\rangle$, and $Y_{k}$.

Now $D\left(Y,|\psi\rangle^{\otimes n}\right)=D\left(\operatorname{Tr}_{A^{\prime} B^{\prime}}|x\rangle\langle x|,| \psi\rangle^{\otimes n}\right)<\epsilon$. Thus, we can use Lemma 7 to show that $\exists|\gamma\rangle \in \mathcal{H}_{A^{\prime}} \otimes \mathcal{H}_{B^{\prime}}$ such that

$$
D\left(|x\rangle,|\psi\rangle^{\otimes n}|\gamma\rangle\right)<2 \epsilon .
$$


Define $\rho=\operatorname{Tr}_{B}|\psi\rangle\left\langle\psi\left|, \quad X=\operatorname{Tr}_{B B^{\prime}}\right| x\right\rangle\langle x|$, and $\Gamma=\operatorname{Tr}_{B^{\prime}}|\gamma\rangle\langle\gamma|$. Since tracing out a subsystem never increases distance between two states

$$
D\left(X, \rho^{\otimes n} \otimes \Gamma\right)<2 \epsilon .
$$

At this point, several different variables have been introduced to label different subsystems of $|x\rangle$. To keep track of their relations to one another, the following diagram may be useful.

$$
\begin{array}{cccc}
\left|x_{k}\right\rangle & \approx|\psi\rangle^{\otimes n} \otimes|\gamma\rangle & \stackrel{\operatorname{Tr}_{A^{\prime} B^{\prime}}}{\longrightarrow} & Y_{k} \approx|\psi\rangle^{\otimes n} \\
& \downarrow \operatorname{Tr}_{B B^{\prime}} & & \downarrow \operatorname{Tr}_{B} \\
X & \approx \rho^{\otimes n} \otimes \Gamma & \stackrel{\operatorname{Tr}_{A^{\prime}}}{\longrightarrow} & \operatorname{Tr}_{A^{\prime}} X \approx \rho^{\otimes n} .
\end{array}
$$

Alice's reduced density matrix $X$ turns out to have a simple expression in terms of $M$ that will make it quite useful to work with. From (12)

$$
\begin{aligned}
\frac{\operatorname{Tr} M M^{\dagger}}{d}|x\rangle\langle x| & =(M \otimes U)\left|\Phi_{d}\right\rangle\left\langle\Phi_{d}\right|\left(M^{\dagger} \otimes U^{\dagger}\right) \\
\frac{\operatorname{Tr} M M^{\dagger}}{d} \operatorname{Tr}_{B B^{\prime}}|x\rangle\langle x| & =M\left(\frac{I_{d}}{d}\right) M^{\dagger}=\left(\frac{1}{d}\right) M M^{\dagger} \\
X=\operatorname{Tr}_{B B^{\prime}}|x\rangle\langle x| & =\frac{M M^{\dagger}}{\operatorname{Tr} M M^{\dagger}} .
\end{aligned}
$$

Thus, $\|X\|=\frac{\left\|M M^{\dagger}\right\|}{\operatorname{Tr} M M^{\dagger}} \leq \frac{1}{\operatorname{Tr} M M^{\dagger}}$. Plugging in (14) gives

$$
\|X\| \leq \frac{2^{c+s}}{d} .
$$

Since Schmidt number can never increase by local operations, we also have that

$$
\operatorname{rank} X \leq d
$$

The proof now follows from (16), (18), and (19) and our results about significant subspaces.

First define $P_{1} \in \mathcal{B}\left(\mathcal{H}_{A}\right)$ to be the projector onto all eigenvectors of $\rho^{\otimes n}$ with eigenvalue of $2^{-n E}$ or greater. There can be no more than $2^{n E}$ such eigenvectors, so $\operatorname{Tr} P_{1} \leq 2^{n E}$. From Lemma 5, we have that

$$
\operatorname{Tr} P_{1} \rho^{\otimes n} \geq \frac{1}{2}-\frac{25 \beta}{\sqrt{n}}>\frac{1}{4}
$$

where the last inequality holds as long as $n_{0}>2500 \beta^{2}$. In terms of significant subspaces, we can summarize this with

$$
\mathcal{S}\left(\rho^{\otimes n}, \frac{1}{4}\right)<\operatorname{Tr} P_{1} \leq 2^{n E} .
$$

We will now use our bound on the rank of $X$ from (19) to show that $\Gamma$ also has a small significant subspace. To accomplish this we will seek constants $\delta_{\rho}$ and $\delta_{\Gamma}$ with the following properties:

- $\delta_{\rho}+\delta_{\Gamma}+\epsilon<1$,

- $\delta_{\Gamma}>4 \epsilon_{0}$,

- $\forall n \geq n_{0}, \mathcal{S}\left(\rho^{\otimes n}, \delta_{\rho}\right)>C 2^{n E+\alpha \sqrt{n}}$ where $n_{0}$ depends on $\rho$ and $C$ does not.

According to Proposition 6, this last condition is met by $\delta_{\rho}=0.95$. To meet the other two, it will suffice to set $\epsilon \leq \epsilon_{0}=0.01$ and $\delta_{\Gamma}=0.04$.
Combining $\delta_{\rho}+\delta_{\Gamma}+\epsilon<1$ with (16) and Proposition 3 yields

$$
\begin{aligned}
\mathcal{S}\left(\rho^{\otimes n} \otimes \Gamma, \delta_{\rho}+\delta_{\Gamma}\right) & <\mathcal{S}\left(\rho^{\otimes n} \otimes \Gamma, 1-\epsilon\right) \\
& \leq \operatorname{rank} X \leq d .
\end{aligned}
$$

Applying Proposition 4 now gives

$$
\begin{aligned}
\mathcal{S}\left(\Gamma, \delta_{\Gamma}\right) & \leq \frac{\mathcal{S}\left(\rho^{\otimes n} \otimes \Gamma, \delta_{\rho}+\delta_{\Gamma}\right)}{\mathcal{S}\left(\rho^{\otimes n}, \delta_{\rho}\right)-1}+1 \\
& <\frac{d}{C 2^{n E+\alpha \sqrt{n}}-1}+1 \\
& \approx \frac{d}{C 2^{n E+\alpha \sqrt{n}}} .
\end{aligned}
$$

In the last line, the factors of +1 and -1 are negligible compared with the exponentials in the numerator and denominator, so we can absorb them into $C$.

Define $P_{2}$ to project onto the highest $\mathcal{S}\left(\Gamma, \delta_{\Gamma}\right)$ eigenvalues of $\Gamma$. Then

$$
\operatorname{Tr} P_{2} \Gamma \geq \delta_{\Gamma} \quad \text { and } \quad \operatorname{Tr} P_{2}=\mathcal{S}\left(\Gamma, \delta_{\Gamma}\right) \leq \frac{d}{C 2^{n E+\alpha \sqrt{n}}} .
$$

Now we combine (18), (21), and (22) to obtain

$$
\begin{aligned}
\operatorname{Tr}\left(P_{1} \otimes P_{2}\right) X & \leq \operatorname{Tr} P_{1} \operatorname{Tr} P_{2}\|X\| \\
& \leq 2^{n E} \cdot \frac{d}{C 2^{n E+\alpha \sqrt{n}}} \cdot \frac{2^{c+s}}{d} \\
& =\frac{2^{c+s}}{C 2^{\alpha \sqrt{n}}} .
\end{aligned}
$$

On the other hand, $P_{1}$ and $P_{2}$ project onto significant subspaces of $\rho^{\otimes n}$ and $\Gamma$, respectively, so

$$
\operatorname{Tr}\left(P_{1} \otimes P_{2}\right)\left(\rho^{\otimes n} \otimes \Gamma\right) \geq \frac{1}{4} \delta_{\Gamma} .
$$

Thus,

$$
\begin{aligned}
2 \epsilon_{0} & \geq 2 \epsilon \geq D\left(\rho^{\otimes n} \otimes \Gamma, X\right) \\
& \geq 2 \operatorname{Tr}\left(P_{1} \otimes P_{2}\right)\left(\rho^{\otimes n} \otimes \Gamma-X\right) \\
& \geq 2\left(\frac{\delta_{\Gamma}}{4}-C^{-1} 2^{c+s-\alpha \sqrt{n}}\right) .
\end{aligned}
$$

Solving for $c+s$ yields

$$
c+s \geq \alpha \sqrt{n}+\log \left(\frac{\delta_{\Gamma}}{4}-\epsilon_{0}\right)-\log C .
$$

So there exist choices of $\alpha, n_{0}, \epsilon_{0}$ that prove the theorem.

Remark: This bound only assumes $O(1)$ error; specifically, $\epsilon_{0}=0.01$. That is, even an entanglement dilution protocol with a nonnegligible amount of loss of fidelity is still covered by Theorem 1, which is, therefore, a rather strong result.

Remark: Suppose we are interested in $o(1)$ error, a stricter requirement. It is not difficult to improve our classical communication bound to $\omega(\sqrt{n})$; by analogy with data compression, achieving error $\epsilon$ can be shown to require inefficiency and classical communication of $\Omega(\alpha \sqrt{n \log (1 / \epsilon)})$. See the Remark just after Proposition 6 for the main modification needed in the proof. 


\section{CONCLUSION AND DISCUSSION}

We have proven that entanglement dilution from any number of singlets to $n$ pairs of bipartite partially entangled pure states necessarily requires $\Omega(\sqrt{n})$ bits of classical communication, thus showing that the main result in [16] is, in fact, optimal.

A technique of our proof is a generalization of the reduction result of [17] in entanglement manipulation protocols. [17] showed that any exact pure state LOCC entanglement manipulation protocol could have two-way communication reduced to one-way communication. We extended their result to protocols ending in mixed states, and proved that Alice can be assumed to transmit her entire measurement outcome with no increase in communication. This reduction applies rather generally and may be useful in deriving bounds on classical communication for other sorts of entanglement manipulations.

An interesting, but difficult, problem is to try to derive similar bounds for transformations from a single copy of one state $\left|\psi_{1}\right\rangle$ to a single copy of another $\left|\psi_{2}\right\rangle$. Here, there are no intermediate results between the constant lower bound of [17] and the construction of [11] and [14] which uses $\log$ rank $\operatorname{Tr}_{B}\left|\psi_{1}\right\rangle\left\langle\psi_{1}\right|$ bits of communication.

A more specific, and perhaps more tractable, problem is that of converting between many copies of different partially entangled states. Suppose we constrain these sorts of interconversions to waste only $o(n)$ bits of entanglement. Recall from [16] that dilution can be performed with $O(\sqrt{n})$ bits of classical communication (and that [14] shows how to reduce this cost by a factor of 2). Our lower bound in Theorem 1 matches this construction up to a constant factor. If we could improve either our lower bound or the protocol of [14], [16] to eliminate this constant factor, then we could prove that, at least for some partially entangled states $\left|\psi_{1}\right\rangle$ and $\left|\psi_{2}\right\rangle$, preparing $\left|\psi_{2}\right\rangle^{\otimes n}$ from $\left|\psi_{1}\right\rangle^{\otimes n \frac{E\left(\psi_{2}\right)}{E\left(\psi_{1}\right)}+o(n)}$ requires $\Omega(\sqrt{n})$ bits of communication.

To see this, consider the following two processes.

Process A: Start from singlets, dilute to $n$ copies of $\left|\psi_{2}\right\rangle$ directly.

Process B: Start from singlets, dilute first to $n \frac{E\left(\psi_{2}\right)}{E\left(\psi_{1}\right)}+o(n)$ copies of $\left|\psi_{1}\right\rangle$ and then apply a conversion procedure from the $n \frac{E\left(\psi_{2}\right)}{E\left(\psi_{1}\right)}+o(n)$ copies of $\left|\psi_{1}\right\rangle$ to $n$ copies of $\left|\psi_{2}\right\rangle$.

From Theorem 1, Process A takes $\Omega\left(\alpha_{\psi_{2}} \sqrt{n}\right)$ bits of classical communication. Process B is a way of realizing Process A and is, thus, constrained by our lower bound. Therefore, it must also take $\Omega\left(\alpha_{\psi_{2}} \sqrt{n}\right)$ bits of classical communication. Now, [14], [16], showed that part 1 of Process B can be done with only

$$
O\left(\alpha_{\psi_{1}} \sqrt{n \frac{E\left(\psi_{2}\right)}{E\left(\psi_{1}\right)}}\right)
$$

bits of classical communication. If the constant factors of Theorem 1 and [14], [16], were the same then the classical communication cost of part 2 of Process B would be lower-bounded by an amount of that is at least the difference between these two numbers, which is positive whenever

$$
\frac{\alpha_{\psi_{2}}}{\sqrt{E\left(\psi_{2}\right)}}>\frac{\alpha_{\psi_{1}}}{\sqrt{E\left(\psi_{1}\right)}} \text {. }
$$

This would establish a total ordering on entangled states. If, instead, we were unable to close the gap between the constants of the upper and lower bounds, then we would have a partial ordering; for some constant $C>1$, converting $\left|\psi_{1}\right\rangle$ to $\left|\psi_{2}\right\rangle$ would require communication whenever

$$
\frac{\alpha_{\psi_{2}}}{\sqrt{E\left(\psi_{2}\right)}}>C \frac{\alpha_{\psi_{1}}}{\sqrt{E\left(\psi_{1}\right)}}
$$

It is an open question whether (26) is a necessary condition for a classical communication bound or whether such a result holds for more general pairs of partially entangled states. Also, unlike the case of diluting from maximally entangled states, there may be a tradeoff between inefficiency and communication when starting with partially entangled states.

We can also apply our main theorem to the resource model of quantum information processing, where it implies that there is a limited extent to which the resource of entanglement can be thought of as independant of form. Thus, when one considers scenarios with prior shared entanglement, one should either a) restrict the scenario to $o\left(n^{2}\right)$ copies of any partially entangled state (if the protocol calls for $O(n)$ bits of classical communication) or b) specify explicitly what forms of bipartite entanglement are allowed.

More generally, the classical communication cost in quantum information processing is an important subject [14]. One rather curious fact about our result is that dilution requires a large amount of classical communication, but there does not appear to be any simple way to use it for signaling. It would be interesting to determine whether any black box capable of performing entanglement dilution could also be used to transmit information, as this would provide an intuitive alternate proof of our main result.

Two final remarks are in order. First, the classical bits transmitted in entanglement dilution constitute classical shared randomness between Alice and Bob. Such classical shared randomness can be an important resource in information processing. However, since those bits are sent through a classical channel, their value is potentially public knowledge. Thus, we can call them shared public randomness. Second, the loss of entanglement in, for example, entanglement concentration will generally give rise to shared randomness that is private to Alice and Bob. Similarly, the loss of entanglement in entanglement dilution will give rise to residual correlations that are almost uncorrelated to the desired final state (according to (15)). Such randomness is private to Alice and Bob, and could, therefore, be used for applications such as a cryptographic one-time-pad. ${ }^{6}$ We believe that a complete theory of the resource model of quantum information processing should take full account of these two resources-shared public randomness and shared private randomness.

After the completion and circulation of a draft version of the current paper, we became aware of the independent proof of almost the same result but with a different approach by Patrick Hayden and Andreas Winter [10].

\footnotetext{
${ }^{6}$ However, Alice and Bob also have the option of keeping the ancillary state quantum, rather than measuring it and generating classical randomness. According to Lemma 7, this ancillary state is nearly separable from the output of the dilution procedure. Thus, Alice and Bob could consider keeping their ancillas and later recycling some of its entanglement.
} 


\section{APPENDIX}

\section{Proof of Lemma 7}

For any density matrices $\rho_{0}$ and $\rho_{1}[8$, eq. (46)] states that

$$
1-F\left(\rho_{0}, \rho_{1}\right) \leq \frac{1}{2} \operatorname{Tr}\left|\rho_{0}-\rho_{1}\right|
$$

where

$$
F\left(\rho_{0}, \rho_{1}\right)=\operatorname{Tr} \sqrt{\sqrt{\rho_{0}} \rho_{1} \sqrt{\rho_{0}}}
$$

is the fidelity. By Uhlmann's theorem [22]

$$
F\left(\rho_{0}, \rho_{1}\right)=\max _{\varphi_{0}, \varphi_{1}}\left|\left\langle\varphi_{0} \mid \varphi_{1}\right\rangle\right|
$$

where $\varphi_{0}$ and $\varphi_{1}$ are purifications of $\rho_{0}$ and $\rho_{1}$, respectively. Equivalently, we can fix an arbitrary purification $\varphi_{0}$ and maximize only over $\varphi_{1}$.

Applying these two results we find that there exists $|\gamma\rangle \in \mathcal{H}_{B}$ such that

$$
\mid\left\langle\psi|(|\phi\rangle \otimes|\gamma\rangle)| \geq 1-\frac{\epsilon}{2}\right.
$$

since we can consider $|\phi\rangle \otimes|\gamma\rangle$ to be a purification of $|\phi\rangle\langle\phi|$.

To obtain the trace distance between these states, write $|\phi\rangle \otimes|\gamma\rangle$ as $a|\psi\rangle+b\left|\psi^{\perp}\right\rangle$, where $|a| \geq 1-\epsilon / 2$ and

$$
|b|=\sqrt{1-|a|^{2}} \leq \sqrt{\epsilon-\epsilon^{2} / 4} .
$$

The trace distance is then given by

$$
\operatorname{Tr}\left|\left(\begin{array}{cc}
1-|a|^{2} & 0 \\
0 & -|b|^{2}
\end{array}\right)\right|=2|b|^{2} \leq 2 \epsilon-\frac{\epsilon^{2}}{2}<2 \epsilon .
$$

\section{ACKNOWLEDGMENT}

The authors wish to thank their colleagues including Charlie Bennett, Andrew Childs, Isaac Chuang, Debbie Leung, Sandu Popescu, Ben Recht, John Smolin, and Jason Taylor for helpful discussions. They are also indebted to Patrick Hayden and Andreas Winter for sharing the draft of [10] and for enlightening discussions.

\section{REFERENCES}

[1] C. H. Bennett, H.-J. Bernstein, S. Popescu, and B. Schumacher, "Concentrating partial entanglement by local operations," Phys. Rev. A, vol. 53, pp. 2046-2052, 1996.

[2] C. H. Bennett, A. W. Harrow, D. W. Leung, and J. A. Smolin, "On the capacities of bipartite Hamiltonians and unitary gates," arXive eprint, quant-ph/0205057, 2002.

[3] E. Boltausen, "An estimate of the remainder in a combinatorial central limit theorem," Z. Wahtscheinlichkeitstheorie, vol. 66, pp. 387-405, 1984.

[4] C. H. Bennett and S. J. Wiesner, "Communication via 1- and 2-particle operators on Einstein-Podolsky-Rosen state," Phys. Rev. Lett., vol. 69, no. 20, pp. 2881-2884, 1992.

[5] H. F. Chau and H.-K. Lo, "Making an empty promise with a quantum computer," Fort. der Phys., pp. 507-520, 1998.

[6] T. M. Cover and J. A. Thomas, Elements of Information Theory. New York: John Wiley and Sons, 1991.

[7] W. Dür, G. Vidal, J. I. Cirac, N. Linden, and S. Popescu. (2001) Entanglement capabilities of non-local Hamiltonians. Phys. Rev. Lett. [Online] vol. 87, no. 137901, arXive eprint quant-ph/0006034.

[8] C. A. Fuchs and J. van de Graaf, "Cryptographic distinguishability measures for quantum mechanical states," IEEE Trans. Inform. Theory, vol. 45, pp. 1216-1227, May 1999.

[9] C. A. Fuchs, "Distinguishability and accessible information in quantum theory," Ph.D. dissertation, Univ. New Mexico, Albuquerque, 1995.

[10] P. Hayden and A. Winter, "On the Communication cost of entanglement transformations," arXive eprint, quant-ph/0204092, 2002.

[11] J. G. Jensen and R. Schack. (2001) A simple algorithm for local conversion of pure states. Phys. Rev. A [Online] vol. 63, no. 062303, arXive eprint quant-ph/0006049.

[12] P. Kaye and M. Mosca, "Quantum networks for concentrating entanglement," arXive eprint, quant-ph/0101009.

[13] H.-K. Lo, "Insecurity of quantum secure computations," Phys. Rev. A, p. $1154,1997$.

[14] - Classical communication cost in distributed quantum information processing-A generalization of quantum communication complexity [Online] vol. 62, no. 012313, arXive eprint quant-ph/99120049.

[15] H.-K. Lo and H. F. Chau, "Is quantum bit commitment really possible?," Phys. Rev. Lett., pp. 3410-3413, 1997.

[16] H.-K. Lo and S. Popescu, "The classical communication cost of entanglement manipulation: Is entanglement an inter-convertible resource?," Phys. Rev. Lett., vol. 83, pp. 1459-1462, 1999.

[17] - Concentrating entanglement by local actions-Beyond mean values [Online] vol. 63, no. 022301, arXive eprint quant-ph/9707038.

[18] D. Mayers, "Unconditionally secure bit commitment is impossible," Phys. Rev. Lett., vol. 78, pp. 3414-3417, 1997.

[19] M. A. Nielsen, "Conditions for a class of entangement transforms," Phys. Rev. Lett., vol. 83, no. 2, pp. 436-439, 1999.

[20] A. Peres, Quantum Theory: Concepts and Methods. Dordrecht, The Netherlands: Kluwer Academic, 1993.

[21] G. Vidal, "Entanglement monotones," J. Mod. Opt., vol. 47, p. 355, 2000.

[22] A. Uhlmann, "The 'transition probability' in the state space of a *-algebra," Repts. Math. Phys., vol. 9, pp. 273-279, 1976. 